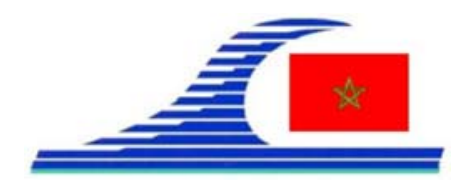

Conférence Méditerranéenne Côtière et Maritime

EDITION 2, TANGER, MAROC (2011)

Coastal and Maritime Mediterranean Conference

Disponible en ligne - http://www.paralia.fr - Available online

\title{
Pressions induites sur le fond au voisinage d'une plaque plane immergée par une houle incidente en profondeur infinie
}

\author{
Julien TOUBOUL ${ }^{1}$, Vincent REY ${ }^{1}$ \\ 1. Université du Sud - Toulon - Var, Institut des Sciences de l'Ingénieur Toulon - Var, \\ LSEET-UMR 6017 CNRS, Av G. Pompidou, BP 56, 83162 La Valette Cedex, \\ France.julien.touboul@univ-tln.fr
}

\section{Résumé :}

Cette étude décrit expérimentalement et analytiquement les effets de pression induits au fond de l'eau au voisinage d'une plaque plane immergée dans le but de réfléchir une houle incidente.

Pour cela, nous considérons une houle monochromatique incidente, qui se propage dans des conditions de "profondeur infinie" en amont de la plaque. L'interaction de cette houle avec la plaque donne naissance à une onde réfléchie, une onde transmise, et une infinité de modes locaux présents au voisinage de la plaque. En comparant l'intensité de la pression au fond avec celle qui serait observée en l'absence de la plaque, nous observons des valeurs jusqu'à trente fois supérieures. Le terme de second ordre, quand à lui, présente également des valeurs très importantes, pouvant parfois dépasser le terme de premier ordre. Ce constat s'explique en termes d'interactions non linéaires entre les différents modes présents.

\section{Mots-clés :}

Pression au fond - Réflexion de la houle - Plaque plane immergée - Couplage de modes non linéaire

\section{Introduction}

En zone côtière, la houle peut être partiellement réfléchie par des variations rapides du fond. Ce "pouvoir de réflexion", lié aux variations de la bathymétrie, a conduit à de nombreuses études et à la réalisation d'ouvrages côtiers immergés destinés à protéger des aménagements ou sites naturels de l'impact de la houle par réflexion partielle de son énergie (voir par exemple REY (1992) et REY (1995)). Pour des zones côtières où la profondeur d'eau croit rapidement, des ouvrages de type plaque immergée ont été proposés pour la protection côtière. Les études de leur pouvoir de réflexion en fonction de la valeur relative de la longueur d'onde de l'onde par rapport à la longueur de la plaque à partir de modèles parfaits et linéaires ont montré la présence de maxima et minima de réflexion (PATARAPANICH,1984; STUROVA, 1991). Ce caractère oscillant de l'intensité de l'énergie réfléchie est dû à un phénomène d'interférence au dessus de la plaque. Les aspects non-linéaires ont fait l'objet d'études plus récentes (BROSSARD \& CHAGDALI, 2001). Les efforts exercés par la houle sur la structure 
La connaissance de la Mer :

un vecteur du développement durable en Méditerranée

ont été détaillés expérimentalement et analytiquement, en s'appuyant sur le modèle utilisé dans cette étude (REY \& TOUBOUL, 2011). Le travail expérimental présenté ici a été réalisé dans le Bassin de Génie Océanique (BGO) FIRST, près de Toulon, France. Il consistait en l'étude de la pression induite au fond par l'interaction entre une houle monochromatique et la plaque plane immergée. La houle considérée se propage, en amont de la plaque, dans des conditions de profondeur infinie. LONGUET-HIGGINS (1950) a montré que pour une onde stationnaire, les effets de pression persistaient indépendamment de la profondeur au second ordre. Ce phénomène est d'autant plus important que la houle est cambrée. Le champ de pression au fond associé pourrait être considéré comme négligeable au premier ordre. Or la dépendance en $\mathrm{z}$ des termes du potentiel des vitesses associés aux modes évanescents, et l'oscillation en bloc au dessous de la structure suggèrent des effets non négligeables au premier ordre. La réflexion partielle de l'onde par la plaque suggère des effets au second ordre. L'étude expérimentale présentée ici avait pour but d'étudier ces pressions au fond, tant au premier qu'au second ordre. Ces résultats sont discutés, et interprétés en termes linéaires ou d'interaction non linéaire entre les différents modes en présence.

\section{Résultats analytiques et expérimentaux}

La houle considérée dans cette étude présente une période $\mathrm{T}=1,4 \mathrm{~s}$. Dans la configuration retenue, la profondeur d'eau est de $\mathrm{h}=3 \mathrm{~m}$. La plaque plane, d'épaisseur $0.10 \mathrm{~m}$ et de longueur $1.53 \mathrm{~m}$, est immergée de telle sorte que sa partie supérieure soit à une profondeur de $0.50 \mathrm{~m}$. Dans ce contexte, le paramètre de profondeur lié à l'onde incidente est $\mathrm{kh}=6,16$, où $\mathrm{k}$ est le vecteur d'onde. La houle incidente considérée se propage donc dans des conditions de profondeur infinie. Trois amplitudes de houles incidentes sont considérées. En termes d'amplitude incidente, les valeurs de $44 \mathrm{~mm}$, $54 \mathrm{~mm}$ et $65 \mathrm{~mm}$ sont analysées. Ces valeurs correspondent respectivement à des cambrures de $\mathrm{ak}=0,09, \mathrm{ak}=0,11$ et $\mathrm{ak}=0,13$.

Le fond du bassin est instrumenté de 16 capteurs de pression, disposés le long de la direction de propagation de la houle, perpendiculairement à la plaque. De cette manière, nous nous ramenons à un problème bidimensionnel. Nous nous intéressons à l'enveloppe du terme de pression oscillant à la fréquence $\mathrm{f}$ de l'onde incidente (terme de premier ordre), ainsi qu'au second harmonique (fréquence $2 \mathrm{f}$, terme de second ordre).

Le calcul théorique au premier ordre est basé sur une formulation intégrale des conditions de continuité de vitesse et de pression aux bords de la plaque. Il suppose un écoulement potentiel, et des ondes de faible amplitude. Il tient compte de la présence de modes évanescents au voisinage de la structure. En ce qui concerne le second ordre, le même problème à la fréquence double est considéré. Seules varient les conditions de forçage, qui ne correspondent plus à une onde incidente, mais à la somme des solutions du problème non linéaire d'ordre 2 dans chaque domaine, faisant intervenir les solutions du problème d'ordre 1 . 
La figure 1 représente l'évolution spatiale du terme de pression, au premier et au second ordre. Dans le référentiel considéré, la plaque s'étend entre $X=6,985 \mathrm{~m}$ et $X=8.515 \mathrm{~m}$. Les symboles $(\bullet, \boldsymbol{\bullet}, \mathbf{\Lambda})$, ainsi que le trait continu correspondent respectivement aux résultats expérimentaux (pour les trois cambrures considérées) et du modèle pour le terme de premier ordre. Les symboles $(+, \times, *)$, ainsi que les traits pointillés, correspondent aux résultats expérimentaux (pour les trois cambrures considérées) et du modèle pour le second ordre. Le terme $\mathrm{P}_{\mathrm{N}}$ utilisé pour la normalisation correspond à l'enveloppe de la pression oscillant à la fréquence fondamentale de la houle, c'est-à-dire à la pression que l'on aurait au fond en présence de la seule houle incidente, et en l'absence de la plaque.

\section{Discussion et conclusion}

La figure 1 illustre la forte augmentation à l'ordre 1 (fréquence f) ainsi que la présence à l'ordre 2 (fréquence $2 \mathrm{f}$ ) de la pression au fond au voisinage de la structure, par rapport à la valeur associée à la houle incidente seule $\left(\mathrm{P} / \mathrm{P}_{\mathrm{N}}=1\right)$. En effet, les pressions mesurées en présence de la plaque immergée peuvent être trente fois supérieures dans son voisinage. Ce résultat est d'autant plus saisissant que la houle considérée se propage en conditions de profondeur infinie, et que l'on aurait pu s'attendre à ce que la perturbation liée à la plaque ne soit pas mesurable au fond. Cette augmentation de la pression au premier ordre est parfaitement décrite par le modèle analytique, laissant entendre qu'il s'agit en partie du rôle joué par les modes évanescents au voisinage de la plaque.

En ce qui concerne le second ordre, nous pouvons constater que là encore, les pressions observées sont bien plus importantes que celles attendues. En effet, les ordres de grandeur en jeux devraient être de l'ordre du carré de la cambrure. Or ils sont ici plusieurs fois supérieurs à l'unité. Cet effet a déjà été décrit par LONGUET-HIGGINS (1950), lorsque deux systèmes de houle de même fréquence se propagent dans des directions opposées. Nous constatons ici que l'interaction non linéaire prévue par Longuet-Higgins dans ce cas de figure est généralisable à l'interaction non linéaire d'un mode propagatif avec des modes locaux. Une conséquence de cette interaction est que le terme de second ordre de la pression au fond peut être d'intensité supérieure au terme de premier ordre.

\section{Remerciements}

Les auteurs remercient le Conseil Général du Var pour son aide financière qui a permis la réalisation des expériences au BGO First en 2010 dans le cadre du GIS HYDRO.

\section{Références bibliographiques}

BROSSARD J., CHAGDALI M. (2001). Experimental investigation of the harmonic generation by waves over a submerged plate. Coastal Engineering, (42), pp 277-290. doi:10.1016/S0378-3839(00)00064-8 
La connaissance de la Mer :

un vecteur du développement durable en Méditerranée

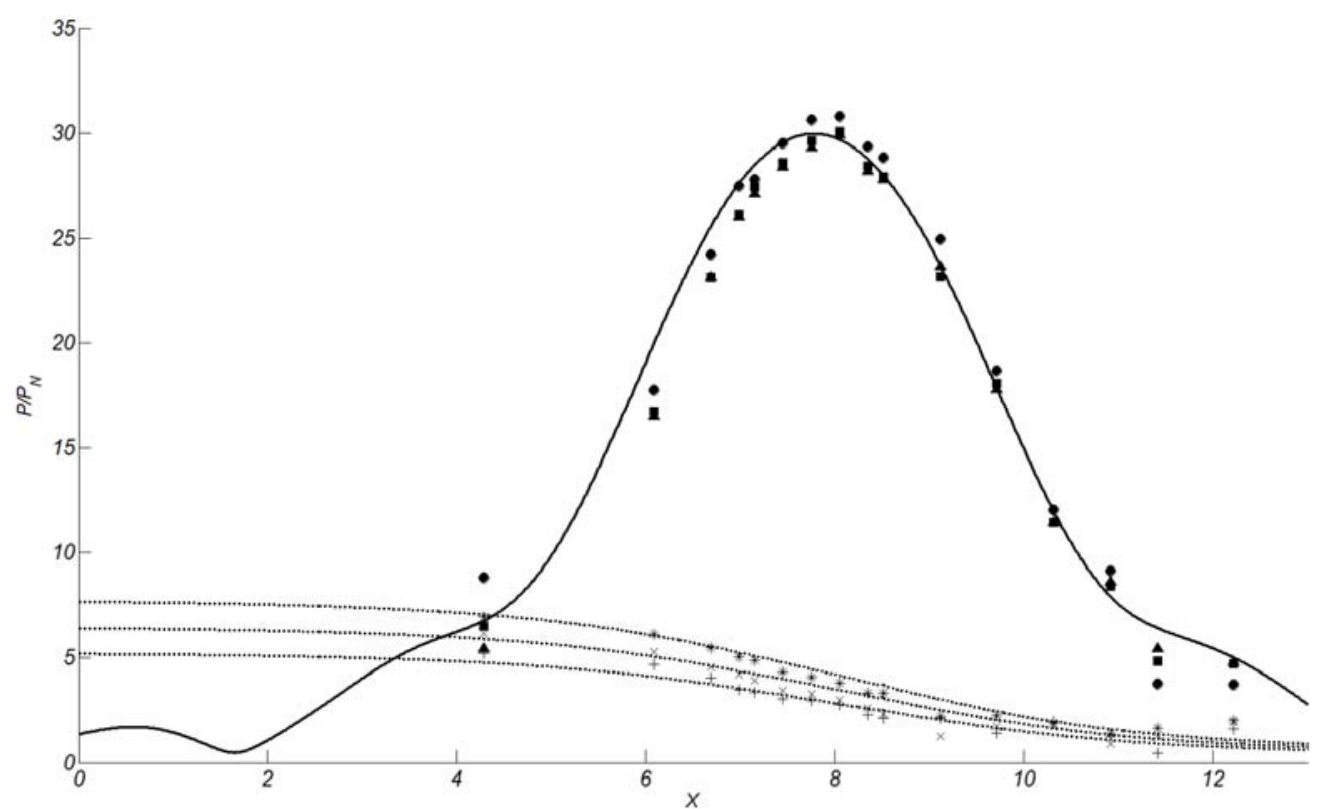

Figure 1. Distribution de la pression au fond, pour les termes de premier ordre

$((\bullet, \mathbf{\square}, \mathbf{\Delta})$ : expérimental, $(-)$ : calculé) et pour les termes de second ordre $((+, \times, *)$ : expérimental, $(. .$.$) : calculé )$.

LONGUET-HIGGINS M.S. (1950). A Theory of the origin of microseisms. Proceedings of the Royal Society, London, Ser. A., (243), pp 1-35.

PATARAPANICH M. (1984). Maximum and zero reflection from submerged plate”. Journal of Waterway, Port, Coastal and Ocean Engineering, (110, 2), pp 171-181.

REY V. (1992). Propagation and local behavior of normally incident gravity waves over varying topography. European Journal of Mechanics B/Fluids, (11), pp 213-232.

REY V. (1995). A note on the scattering of obliquely incident surface gravity waves by cylindrical obstacles in waters of finite depth. European Journal of Mechanics B/Fluids, $(14,1)$, pp 207-216.

REY V., BELZONS M., GUAZZELLI E. (1992). Propagation of surface gravity waves over a rectangular submerged bar. J. Fluid Mech., (235), pp 453-479. doi:10.1017/S0022112092001186

REY V., TOUBOUL J. (2011). Forces and moment on a horizontal plate due to regular and irregular waves in the presence of current. Applied Ocean Research, $(33,2)$, pp 88-99.

STUROVA V. (1991). Propagation of plane surface waves over an underwater obstacle and a submerged plate. Journal of applied mechanics and technical physics, $(32,3)$, pp 453-479. 